\title{
Mining Daily Work Report Data for Detecting Patterns of Construction Sequences
}

\author{
K. Joseph Shrestha ${ }^{\mathrm{a}}$, Chau Le ${ }^{\mathrm{b}, *}, \mathrm{H}$. David Jeong ${ }^{\mathrm{b}}$, and Tuyen $\mathrm{Le}^{\mathrm{c}}$ \\ ${ }^{a}$ Department of Engineering, Engineering Technology, and Surveying, East Tennessee State University, Johnson City, TN 37614, United States \\ ${ }^{b}$ Department of Construction Science, Texas A\&M University, College Station, TX 77843, United States \\ ${ }^{c}$ Glenn Department of Civil Engineering, Clemson University, Clemson, SC 29634, United States
}

\begin{abstract}
Sequencing construction activities in highway projects is a complex planning process which requires not only considerable knowledge and practical experience of the planner/scheduler about various relevant aspects, such as the activities themselves, construction and procurement processes, and construction methods, but also input from other key members of the project regarding specific constraints and requirements. Moreover, sequencing is an iterative process; the sequence developed in the planning phase is likely to change in the construction phase. Therefore, learning from as-built schedules of past completed projects is needed to improve the planning and scheduling processes for future projects. In current practices, most state Departments of Transportation (DOTs) still mainly rely on schedulers' experience for schedule development. A data-driven systematic approach is still lacking, although the highway agencies have been spending a significant amount of money, time, and effort to collect various digital data during the construction process. This study aims to leverage historical digital daily work report data available in the DOTs' database to detect patterns of construction sequences in highway projects. Daily work report data collected from a state DOT were used to conduct a case study that developed a Sequential Pattern Mining algorithm to extract frequent sequential relationships among the activities for one major type of highway projects.
\end{abstract}

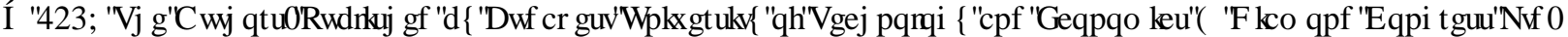

Peer-review under responsibility of the scientific committee of the Creative Construction Conference 2019.

Keywords: Activity sequencing; Construction sequences; Daily work report data; Project scheduling; Sequential pattern mining.

\section{Introduction}

Schedule development is an essential component of construction planning that have a critical impact on successful project delivery $[1,2]$. An optimized schedule enables the contractors and owners to complete a project on time with the minimum resources [3]. Schedules are a valuable tool to support project stakeholders in communicating and coordinating construction activities, track construction progresses after construction starts, and analyze and quantify the potential impact of delays on the overall project schedule. Developing a realistic schedule is challenging for inexperienced as well as experienced schedulers [4]. It is a complex process and requires knowledge of construction methods, materials, and labor productivity [5]. An as-planned schedule development typically consists of three steps: a) identification of activities through work breakdown structure development for the given project, b) determination of the duration of each activity, and c) determination of a logical and realistic sequence of activities [4]. Many academic 
Shrestha, Le, Jeong, and Le / Proceedings of the Creative Construction Conference (2019) 079 https://doi.org/10.3311/CCC2019-079

studies have focused on the first two steps: a) identifying construction activities and b) determining activity durations using historical and reliable production rates to enhance the accuracy of schedule development $[6,7]$. Regarding determining the sequence of activities, many studies pointed out the heavy reliance of owners and contractors on the experience and knowledge of experienced schedulers as the single most crucial source [5, 8].

In the highway industry sector, some Departments of Transportation (DOTs) such as Oklahoma DOT and Texas DOT have developed a set of scheduling templates that store a typical sequence of activities for a specific type of project to facilitate their scheduling activities. This approach helps capture and take advantage of experience schedulers' knowledge even after their retirement. However, such an approach has at least three significant limitations. First, it relies heavily on the experience of a scheduler to develop such templates. Second, various types of projects may have different construction sequences; multiple templates will need to be developed manually, one for each project type. Third, construction means and methods may evolve, and such static sequencing templates may become outdated.

In addition to these schedule templates, if there is a systematic approach to discovering the knowledge of precedence relationships of activities from historical projects and having the knowledge available for schedulers, it would greatly empower schedulers in gaining confidence in developing a new project's schedule and also help an inexperience scheduler to develop a schedule with substantial evidence. More than thirty DOTs have started to use digital Daily Work Reports (DWRs) which contain rich project progress and performance data at the construction activity level. This digital dataset can be directly used to discover various sequences of construction activities when appropriate data analytics is employed. This study applies a powerful Sequential Pattern Mining (SPM) algorithm to readily available historical DWR data to discover precedence relationships of activities.

\section{Background}

\subsection{Prior studies}

Prior studies suggest that schedules are mostly developed manually, especially in activity sequence development [6]. Existing studies on generating activity sequences have been focused on utilizing the expertise of experienced schedulers or logical assumptions about construction activity sequences. For example, Jeong et al. [8] developed fourteen different highway scheduling templates based on the Oklahoma DOT schedulers' experience. Bruce et al. [5] utilized a list of controlling activities and developed templates based on experts' inputs for 12 types of road and bridge construction projects. They also studied the resident engineers' project diaries and other project documentation to develop the templates.

In the vertical construction, some studies that were conducted to improve scheduling practices can be conceptually adopted to the horizontal construction. Cherneff et al. [1] developed a systematic approach to generating activity sequences by assigning various component constraints, i.e., physical constraints based on the construction components. An example of such component constraint is that a door can only exist in a wall. Therefore, a wall must be constructed before a door is installed. Echeverry et al. [2] used the similar approach with four types of logical assumptions: a) physical relationships between building components, b) interaction of construction trades, c) interference-free path for the movement of construction equipment and materials, and d) safety considerations. Similarly, Fischer and Aalami [4] generated component-constrained and activity-constrained relations. In a study by Kim et al. [6], identification of construction activities was improved by utilizing Building Information Models (BIMs), but activity sequencing was still based on a set of static sequencing templates similar to the previous studies. Thus, existing studies have a limitation of being static and being dependent on the knowledge of experienced schedulers.

\subsection{Daily work report data}

DOTs, particularly resident engineers and site inspectors, collect a significant amount of data on highway projects, such as construction activities, labor hours, types of equipment used, equipment hours, and weather data. Currently, more than thirty DOTs in the U.S. are using digital DWR systems. Fig. 1 shows a screenshot of the AASHTOWare 
Shrestha, Le, Jeong, and Le / Proceedings of the Creative Construction Conference (2019) 079 https://doi.org/10.3311/CCC2019-079

SiteManager, which is the most popular DWR system among DOTs. Apart from being mainly used for payment and documentation purposes, DWR systems have potentials to be applied in other applications such as as-built schedule development and construction sequence determination. However, most SHAs have not benefited from those potential applications possibly because of the lack of knowledge on those potential benefits, enabling methodologies, and automation processes.

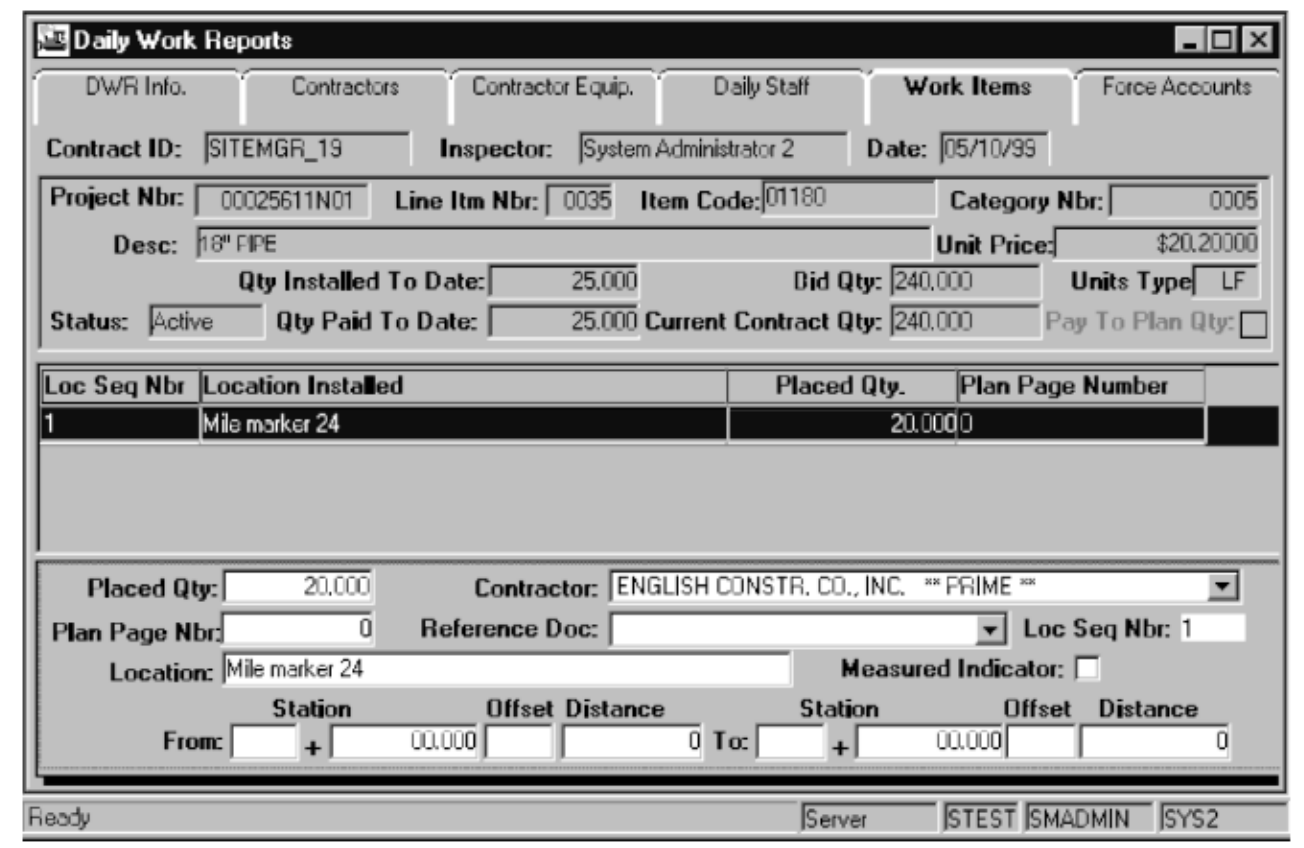

Fig. 1. AASHTOWare SiteManager screenshot

DWR data attributes are linked to separate work items. DOTs have developed an extensive list of work items that are primarily developed to facilitate the bidding process under the unit cost contracting method. Those work items are also used to develop a project schedule since they are independent work activities. DOTs have also developed and maintained specifications that provide a detailed description of each work item. For example, an item code "01180" in Fig. 1 indicates a work item "supply and installation of a mile marker." A typical set of data attributes collected in DWR systems can be classified into six categories: general information, work activities, weather information, equipment, labor, and remarks. Among these six categories, the category of work activities was used for this study. The start dates of various activities can be extracted from the DWR system and then compared to identify the activity sequences for all historical projects. Those activity sequences can be identified using SPM algorithms.

\subsection{Sequential pattern mining}

A SPM algorithm can help identify interesting and hidden sequential patterns from a set of sequential data [9]. SPM algorithms have been used for various applications, such as DNA sequencing, medical treatment, consumer behavior, web access pattern, and stock market [9-11]. For example, if a consumer buys a cell phone on an e-commerce website, a case for the phone is likely to be recommended to the consumer.

In identifying the sequences of construction activities, as-built schedules of past projects can be analyzed using a SPM algorithm to help determine the sequences of activities for a new project. The Sequential Rules Common to Several Sequences (CMRules) is an open source algorithm that was used for this study [10]. The CMRules algorithm finds the sequential patterns that frequently appear in a sequence database and meet a minimum threshold value of confidence and support. For a rule in the form of $X->Y$ (Activity $Y$ happens after activity $X$ happens), the confidence and support of the rule are defined as the following. The support of the rule is the number of sequences containing the activity $X$ 
Shrestha, Le, Jeong, and Le / Proceedings of the Creative Construction Conference (2019) 079 https://doi.org/10.3311/CCC2019-079

before activity $Y$ in the sequence database [10]. The relative support of the rule is the support divided by the number of sequences in the sequence database [10]. The confidence of the rule is the ratio between the support and the number of sequences containing activity $X[10]$.

\section{Mining DWR data for detecting patterns of construction sequences}

\subsection{The proposed framework}

This study proposes a framework for mining DWR data to detect patterns of construction sequences. The framework consists of six components: a) database preparation, b) project type selection c) data extraction, d) data transformation, and e) application of CMRules. First, DWR data was obtained from a current DWR system. As different types of projects may have different project sequencing, DWR data were separated based on project types. For a specific project type, relevant DWR data were selected. Start date information on each activity was extracted for each selected project. The data were then transformed into a format suitable for applying CMRules algorithm. CMRules identified and generated precedence relationships found in the DWR data.

\subsection{Case study}

A DWR database was obtained from a DOT in the Microsoft Access format. The database consisted of project information on over 2,000 projects from 2001 to 2014 . Table 1 presents the top five project work types in terms of the total dollar amount. The project type "widening existing roadway" was the largest work type of all. This type includes the addition of passing lanes to improve traffic flow and road safety conditions. It also had a large number of projects. The work type "widening existing roadway" was then selected for illustrating the framework.

Table 1. Top five project work types in terms of the total dollar amount

\begin{tabular}{llll}
\hline Work type code & Work type & Total dollar amount & Number of projects \\
\hline 04 & Widening existing roadway & $1,799,474,488$ & 204 \\
19 & Structures and approaches & $864,634,085$ & 231 \\
07 & Overlay & $806,625,107$ & 1264 \\
06 & Rehabilitation & $605,243,188$ & 49 \\
16 & Grading and structures & $482,557,003$ & 42 \\
\hline
\end{tabular}

Since there were 204 projects (see Table 1), the sequence database had 204 sequences. Each sequence was the list of work items ordered sequentially based on the extracted start time information from the DWR system. The CMRules algorithm was applied to extract sequential patterns with various values of minimum relative support and confidence. The number of discovered patterns is shown in Table 2.

Table 2. Number of patterns obtained with different values of support and confidence

\begin{tabular}{llll}
\hline Relative support & \multicolumn{2}{l}{ Confidence } & \\
\cline { 2 - 4 } & 0.9 & 0.8 & 0.7 \\
\hline 0.6 & 2 & 9 & 9 \\
0.5 & 2 & 220 & 643 \\
0.4 & 290 & 6,412 & 12,643 \\
\hline
\end{tabular}


Shrestha, Le, Jeong, and Le / Proceedings of the Creative Construction Conference (2019) 079 https://doi.org/10.3311/CCC2019-079

Two of the results obtained from the analysis are presented below, including a simple one with two items (i.e., one-toone relation) and a complex one with multiple items (many-to-many relation).

- (603001 -> 412001), 117, 0.78: This sequential rule indicates that the work item 603001 (maintenance of traffic) starts before the work item 412001 (cold milling asphalt pavement) in 117 projects (support). In the remaining projects, either one of those items is not included in the same project, or work item 412001 starts at the same time or before the item 603001. In 78\% of the projects that include the work item 603001, the work item 412001 follows item 603001. Based on this sequential rule, if a new project includes the two items, the item 412001 would be recommended to start before the item 603001 .

- $(210201,303107,604023 \Rightarrow 624001,719001,719101), 112,0.78$ : This many-to-many relationship includes three preceding work items followed by three work items. The three preceding items can start any time relative to each other; the three succeeding items can also start anytime relative to each other. The three preceding items are 210201 (unclassified excavation), 303107 (aggregate base course (class 7)), and 604023 (traffic drums); the three succeeding items are 624001 (solid sodding), 719001 (thermoplastic pavement marking, white-100), and 719101 (thermoplastic pavement marking, yellow-100). The three preceding items occurred before the succeeding items in 112 projects. Further, in $78 \%$ of the projects containing the preceding items, the succeeding items listed above start after the preceding items.

One-to-one sequences are visualized in a chart that can be used to visually extract the activity sequences for a new project (Fig. 2). The chart shows the work items as nodes and sequences by arrows similar to an activity precedence diagram. For example, regarding the first sequential rule discussed above, activity 603001 (maintenance of traffic) and activity 412001 (cold milling asphalt pavement) are highlighted in red in Fig. 2. The sequencing of two activities is indicated by the arrow connecting them $(603001 \Rightarrow 412001)$. The widths of the arrow lines indicate the confidence of the sequence: the thicker the arrow line is, the higher the confidence is. Based on the activities of a new project, a relevant portion of this chart can be extracted visually to develop activity precedence diagram for the project.

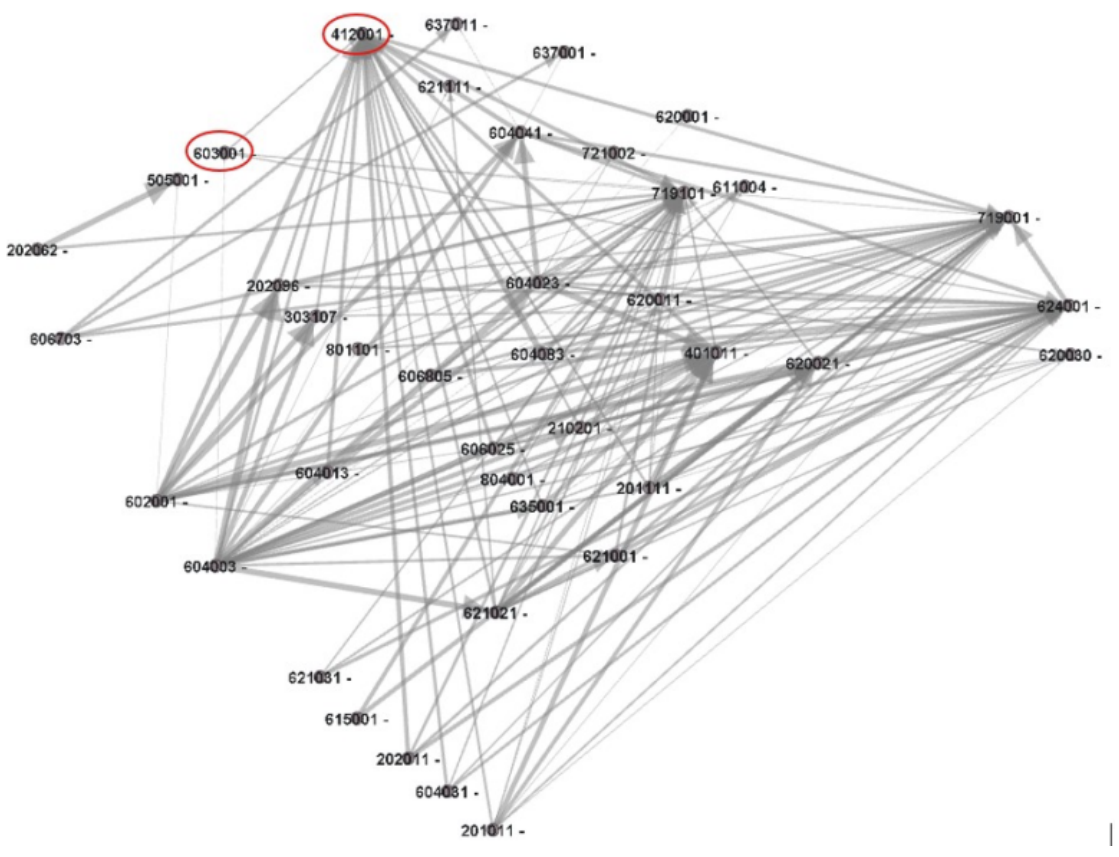

Fig. 3. Visualization of precedence relationships of activities 
Shrestha, Le, Jeong, and Le / Proceedings of the Creative Construction Conference (2019) 079

https://doi.org/10.3311/CCC2019-079

\section{Conclusion}

While some industries have heavily utilized their data to obtain data-driven insights, the construction industry is lagging behind. This study developed a methodology to discover precedence relationships of activities based on DWR data collected by DOTs. Currently, state DOTs and contractors rely on engineers' experience to develop activity precedence diagram, which is very time consuming and complex. In this study, a novel framework based on the SPM algorithm was developed to automate such a process. This framework will aid schedulers in quickly identifying precedence relationships of activities, which is the most complicated part of schedule development. Thus, it provides guidance to the schedulers based on the historical data, minimizes their inputs, and improves their confidence. A dataset from a DOT was analyzed to illustrate the framework. The SPM algorithm generated 12,643 sequential rules that become a knowledge base to generate activity precedence diagram for new projects. However, this study only identified the start-to-start relationships among activities. Further research will be conducted to explore other relationship types such as finish-to-start and finish-to-finish relationships.

\section{References}

1. Cherneff, J., R. Logcher, and D. Sriram, Integrating CAD with Construction - Schedule Generation. Journal of Computing in Civil Engineering, 1991. 5(1): p. 64-84. https://doi.org/10.1061/(ASCE)0887-3801(1991)5:1(64)

2. Echeverry, D., C.W. Ibbs, and S. Kim, Sequencing Knowledge for Construction Scheduling. Journal of Construction Engineering and Management, 1991. 117(1): p. 118-130. https://doi.org/10.1061/ (ASCE)0733-9364(1991)117:1(118)

3. Jaskowski, P. and A. Sobotka, Multicriteria construction project scheduling method using evolutionary algorithm. Operational Research, 2006. 6(3): p. 283-297. https://doi.org/10.1007/ BF02941257

4. Fischer Martin, A. and F. Aalami, Scheduling with Computer-Interpretable Construction Method Models. Journal of Construction Engineering and Management, 1996. 122(4): p. 337-347. https:// doi.org/10.1061/(ASCE)0733-9364(1996)122:4(337)

5. Bruce, R.D., et al., An Expert Systems Approach to Highway Construction Scheduling. Technology Interface International Journal, 2012. 13(1): p. 21-28.

6. Kim, H., et al., Generating construction schedules through automatic data extraction using open BIM (building information modeling) technology. Automation in Construction, 2013. 35: p. 285-295. https://doi.org/10.1016/j.autcon.2013.05.020

7. Woldesenbet, A., D.H.S. Jeong, and G.D. Oberlender, Daily Work Reports-Based Production Rate Estimation for Highway Projects. Journal of Construction Engineering and Management, 2012. 138(4): p. 481-490. https://doi.org/10.1061/(ASCE)CO.1943-7862.0000442

8. Jeong, H.S., et al., Automated contract time determination system for highway projects. Automation in Construction, 2009. 18(7): p. 957-965. http://dx.doi.org/10.1016/j.autcon.2009.04.004

9. Chand, C., A. Thakkar, and A. Ganatra, Sequential pattern mining: Survey and current research challenges. International Journal of Soft Computing and Engineering, 2012. 2(1): p. 185-193.

10. Fournier-Viger, P., et al., A survey of sequential pattern mining. Data Science and Pattern Recognition, 2017. 1(1): p. 54-77. https://doi.org/10.1145/3314107

11. Mooney, C.H. and J.F. Roddick, Sequential pattern mining -- approaches and algorithms. ACM Computing Surveys, 2013. 45(2): p. 1-39. https://doi.org/10.1145/2431211.2431218 